\title{
Practical Learning Research at Scale
}

\author{
Ken Koedinger \\ Professor of Human Computer Interaction and Psychology \\ Director, Pittsburgh Science of Learning Centre \\ Carnegie Mellon University, USA \\ koedinger@cmu.edu
}

\begin{abstract}
Massive scale education has emerged through online tools such as Wikipedia, Khan Academy, and MOOCs. The number of students being reached is high, but what about the quality of the educational experience? As we scale learning, we need to scale research to address this question. Such learning research should not just determine whether high quality has been achieved, but it should provide a process for how to reliably produce high quality learning. Scaling practical learning research is as much an opportunity as a problem. The opportunity comes from the fact that online courses are not only good for widespread delivery, but are natural vehicles for data collection and experimental instrumentation. I will provide examples of research done in the context of widely used educational technologies that both contribute interesting scientific findings and have practical implications for increasing the quality of learning at scale.
\end{abstract}

\section{BIOGRAPHY}

Dr. Ken Koedinger is Professor of Human-Computer Interaction and Psychology at Carnegie Mellon. His research has contributed new principles and techniques for the design of educational software and has produced basic cognitive science research results on the nature of student thinking and learning. Koedinger directs LearnLab (learnlab.org), started with 10 years of NSF Center funding and is now the scientific arm of the Simon Initiative (cmu.edu/simon). LearnLab has created a social-technical infrastructure to support data-driven investigations in education and, more generally, to leverage collaborations of scientists and practioners toward understanding the conditions that cause robust student learning. Koedinger's publications include the

- Knowledge-Learning-Instruction Framework http://pact.cs.cmu.edu/pubs/KLIKoedingerCorbettPerfetti2012-pre.pdf

- Cognitive Science, 2012) and Instructional Complexity http://pact.cs.cmu.edu/pubs/KoedingerScience-2013.pdf (Science, 2013)

See http://pact.cs.cmu.edu/koedinger.html for more.

\footnotetext{
Permission to make digital or hard copies of part or all of this work for personal or classroom use is granted without fee provided that copies are not made or distributed for profit or commercial advantage, and that copies bear this notice and the full citation on the first page. Copyrights for third-party components of this work must be honored. For all other uses, contact the owner/author(s). Copyright is held by the author/owner(s).

L@S 2016, April 25-26, 2016, Edinburgh, UK.

ACM 978-1-4503-3726-7/16/04

http://dx.doi.org/10.1145/2876034.2876054
} 